Research Article

\title{
Somatic Self-Compassion Training Reduces Perceived Stress, Internalized Shame, and Bodily Shame while Increasing Coping Self- efficacy and Self-compassion
}

\author{
Sara B Taylor ${ }^{1,}{ }^{*}$, Kristy Arbon ${ }^{2}$, Faith Reynolds ${ }^{1}$
}

1. Psychology Department, Hendrix College, 1600 Washington Ave, Conway, AR 72032, United States; E-Mails: taylor@hendrix.edu; reynoldsfa@hendrix.edu

2. HeartWorks Training LLC, 7217 Watson Road, PO Box 190443 St Louis, MO 63119, United States; E-Mail: kristy@heartworks.training

* Correspondence: Sara B Taylor; E-Mail: taylor@hendrix.edu

Academic Editor: Viann N. Nguyen-Feng

Special Issue: Yoga and Mindfulness

$O B M$ Integrative and Complementary Medicine 2021, volume 6 , issue 1

doi:10.21926/obm.icm.2101011
Received: October 27, 2020

Accepted: March 23, 2021

Published: March 31, 2021

\begin{abstract}
Somatic Self-Compassion ${ }^{\circledR}$ training is a newly developed trauma-informed self-compassion training that was designed to combine interoception and sensory modulation in order to teach individuals to more effectively cope with current and past stress. Thirty-two nonclinical adults across five cohorts participated in this pre-post design feasibility study. Participation in Somatic Self-Compassion ${ }^{\circledR}$ Online resulted in significant reductions in perceived stress, internalized shame, and bodily shame and significant improvements in coping self-efficacy and self-compassion. Of particular importance for moving the field of self-compassion research forward is the strengthened case for inclusion of shame as a dependent variable in order to understand the relationship between self-compassion, shame, and stress.
\end{abstract}

\section{Keywords}

Self-compassion; somatic; stress; shame; trauma

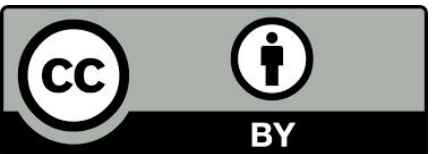

(C) 2021 by the author. This is an open access article distributed under the conditions of the Creative Commons by Attribution License, which permits unrestricted use, distribution, and reproduction in any medium or format, provided the original work is correctly cited. 


\section{Introduction}

Stress is an increasingly pervasive concern within our society and it carries with it many longlasting and detrimental effects. Stress significantly affects all biological systems and is associated with numerous health issues from depression and diabetes $[1,2]$ to impairments in cognitive functioning [3, 4]. Traumatic stress is also more prevalent than commonly thought. According to national estimates, $87.9 \%$ of Americans have been involved in traumatic experiences based on DSM-5 diagnostic criteria and $8.3 \%$ of these go on to develop post-traumatic stress disorder (PTSD) in their lifetime [5]. Given the widespread exposure to traumatic and non-traumatic stress, it is imperative to develop skills to cope adaptively.

The ability to respond to stress or cope adaptively is often something that needs to be learned. In the absence of adaptive coping skills, many individuals fall into unhealthy habits such as excessive caffeine or alcohol consumption or overworking in an attempt to temporarily relieve their stress [6, 7]. However, increasing body awareness and self-compassion during difficult experiences effectively reduces the negative impacts of stress $[8,9]$. To address rising needs for adaptive coping skills, Somatic Self-Compassion ${ }^{\circledR}$ (SSC) training was recently developed to bring together self-compassion and body awareness - via interoception and sensory modulation - in order to teach individuals to more effectively cope with current and past stress and trauma.

While there are several ways to improve body awareness and coping that is supportive of physiological and emotional needs, improving interoceptive awareness and sensory modulation skills are particularly promising. Interoception is the awareness individuals have of their body's internal physical and emotional states, which can help them determine the appropriate response to return to equilibrium through, amongst other possible responses, sensory modulation [10, 11]. Sensory modulation interventions involve recruiting sensory experiences to soothe and bring the nervous system back into balance, leading to better emotion-regulation [12, 13]. Individuals who have lower interoceptive awareness have more difficulty with efficient self-regulation and problem-solving, which can lead to significant and negative impacts on one's health and wellbeing [14]. Consequently, interoceptive awareness, as well as the ability to skillfully respond to stress, is critical for promoting healthy coping behaviors.

Self-compassion practices are also associated with increases in psychological wellbeing [15]. For example, greater self-compassion is associated with adaptive coping ability during times of stress by lowering cortisol levels and increasing heart-rate variability [16]. Similarly, Breines et al. (2014) [17] found that participants with higher levels of self-compassion had significantly lower levels of stress-induced inflammation compared to individuals who reported lower self-compassion. Selfcompassion helps individuals acknowledge that their negative emotions are important and valid and reduces the experience of shame for feeling these emotions [18]. Recent findings also indicate that individuals participating in self-compassion programs experience a reduction of traumarelated shame [19]. This is important as shame appears to be common among those who have experienced trauma and it can prolong the symptoms of some forms of PTSD $[19,20]$.

The concept of self-compassion involves four components: (a) an awareness of one's own suffering, (b) sympathetic concern related to being emotionally moved by one's own suffering, (c) 
a wish to relieve one's own suffering, and (d) a responsiveness or readiness to take action to relieve one's own suffering [21]. Training in self-compassion can be done in a number of different ways, generally relying on inviting repetition of particular thoughts and gestures, along with the positive reinforcement of a group of fellow participants.

The SSC program invites participants to develop three skill sets that reflect the four components of self-compassion. The first skill set, Affectionate Awareness, is developed through offering kindness toward oneself in meditations and through reflection exercises and group discussions that connect participants with their strengths, values, motivations, and goals. Courageous Connection, the second skill set, is developed through learning why our body responds in particular ways to our external world, making meaning of the stress response, exploring neurochemicals, validating the protective stress response cycle, and identifying links between past experiences, values, and present moment responses. The final skill set, Radical Response, is cultivated when participants learn new ways to tend to stress like validating rather than judging experience, using soothing and supportive gestures toward oneself, and developing opportunities for flow and self-care.

SSC is similar to other programs that train in self-compassion, such as Mindful Self-Compassion [18], Compassion Cultivation Training [21], and Compassionate Mind Training [22] in that it is a scaffolded, mindfulness-based program that runs over a number of weeks and it includes talks by a teacher, contemplative practices, interactive and personal exercises, and discussions. SSC is most similar to MSC as it trains specifically in self-compassion skills while the other two programs train in skills of both compassion toward others and compassion toward self. Beyond the somatic focus, SSC differs from these programs in that the origins and focus are implicitly and explicitly secular rather than based on Buddhist psychology. SSC training also differs from the other three programs in that it spends several weeks developing the motivation to practice before practicing. This is an important component that tends to be missing from other self-compassion trainings [23]. It is also specifically trauma-informed using the Substance Abuse and Mental Health Services Administration [24] guidelines, and aims to destigmatize trauma and help participants understand trauma through the lens of evolutionary science, neurophysiology, and common humanity.

The goal of the present study is to evaluate the effectiveness of online SSC training in improving psychological and affective wellbeing by reducing individuals' perceived stress, while increasing coping self-efficacy and self-compassion. In addition, because of the focus on somatic intelligence (e.g., interoception and sensory modulation) and the normalization of the effects of trauma, we anticipated that SSC training would significantly reduce both internalized (trait) and bodily shame. We also predicted that motivation to practice self-compassion would increase as a result of the training and would correlate with changes in our primary dependent variables. Measures were assessed across multiple cohorts at the beginning and end of SSC training.

\section{Materials and Methods}

All procedures were determined to be exempt from full review by our institutional Human Subjects Review Board. Confirmation of this exemption was received on January 16, 2019. Our institutional HSRB ensures that human subjects research complies with the regulations of the United States Department of Health and Human Services for the Protection of Human Research Subjects (Part 46 of Title 45 of the Code of Federal Regulations, as amended) and with the 
principles set forth in the Report of the National Commission for Protection of Human Subjects of Biomedical and Behavioral Research, entitled Ethical Principles and Guidelines for the Protection of Human Subjects of Research (also known as the Belmont Report).

\subsection{Participants}

Thirty-two participants who voluntarily enrolled in SSC training completed all parts of this study. Participants were included across five cohorts $(M=6.4$, range $=3-11$ participants/cohort) over a 14-month period. Ages were captured as ranges, with one participant in 18-24 years, 10 in 35-44 years, 9 in 45-54 years, 8 in 55-64 years, and 4 in 65+ years. Gender was distributed as 29 females, 3 males, and 0 non-binary and transgender. Participants were recruited via email during the first two weeks of the training and the incentive was access to a pre-recorded SSC workshop of their choice (worth \$25) if participants completed both pre and post-test questionnaires. Data was collected anonymously online using a password-protected Survey Monkey account, and participants self-generated IDs based on unchanging personal information for pre-post comparisons.

\subsection{Intervention}

Participants completed the thirteen-week online SSC program in which they learned methods to better prepare them to practice self-compassion (See Figure 1 for a timeline of this program). The first segment is a four-week block designed for individuals to learn about self-compassion and motivate them through their strengths and values to use it. The second segment is a five-week block designed to help individuals be in better communication with their body and its needs, introducing interoceptive and sensory modulation practices for self-soothing coping mechanisms in times of stress. The second segment also focuses on developing an understanding of the body's stress response system, especially how the autonomic nervous system protects us from the effects of traumatic stress. The third segment concludes the last four weeks of the program and helps individuals develop habits that allow them to practice the learned techniques beyond their time in SSC training. Each week participants received pre-work to read, had the opportunity to join a 2hour online instructor-facilitated video training (including a discussion and practice), and discussed material and experiences on the discussion board. Participants also received recommended practices and further reading to integrate the material for each week. The instructor remained the same across all five cohorts that completed this training. 


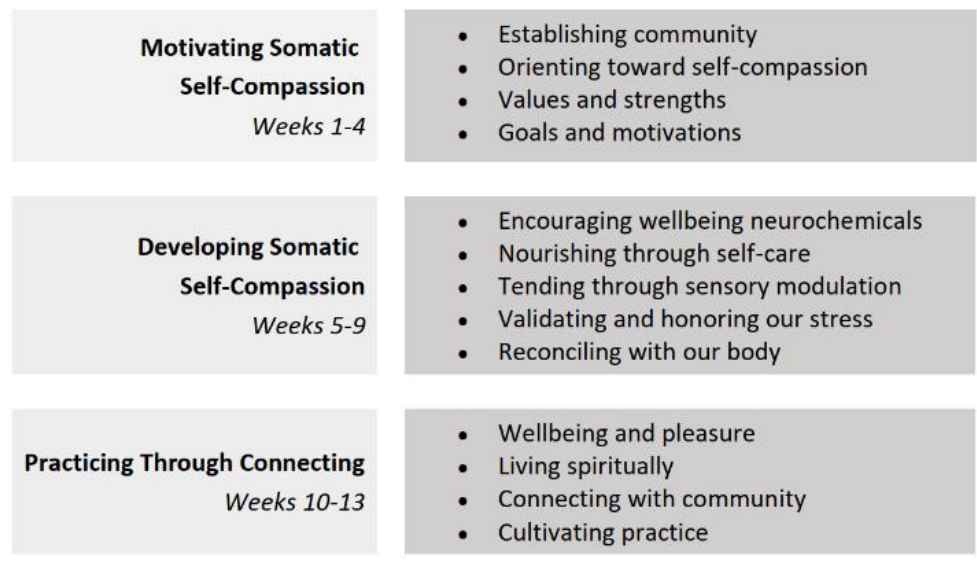

Figure 1 Somatic Self-Compassion online training timeline and modules.

\subsection{Assessments}

Participants completed a baseline self-report questionnaire (time 1) online during the second or third week of the program and a post-intervention questionnaire (time 2 ) online during the last week of the program and up to two weeks afterward.

\subsubsection{Stress}

The Perceived Stress Scale [25] was used to measure how stressful participants perceived their life to be over the last month. The internal reliability for this scale was $\alpha=.89$.

\subsubsection{Shame}

The Internalized Shame Scale [26] was used to measure the frequency of intense negative emotions and cognitions of shame that are internalized to participants' sense of self. The internal reliability for this scale was $\alpha=.95$. Self-esteem was measured using the shortened version of the Rosenberg Self-Esteem Scale [27]. This scale is included in the ISS as the only positively worded items in order to prevent response bias [26]. Consequently, this measure is reported in the results but not presented in the tabular results or discussion. The internal reliability for this scale was $\alpha$ $=.92$.

\subsubsection{Bodily Shame}

Feelings of shame about the body were measured using the subset of four questions from the Experience of Shame Scale $[28,29]$. The internal reliability for this scale was $\alpha=.93$.

\subsubsection{Coping Self-Efficacy}

Perceived ability to cope with challenging situations or stressors was measured with Coping Self-Efficacy Scale [30]. The internal reliability for this scale was $\alpha=.96$.

\subsubsection{Self-Compassion}


The Self-Compassion Scale [31] was used to measure self-compassion. The subscales assessing the positive (self-kindness, common humanity, and mindfulness) and negative (self-judgment, isolation, and over-identification) aspects of the three components of self-compassion were also used. The internal reliability for this scale was $\alpha=.92$

\subsubsection{Motivation}

As an initial inquiry into how aspects of motivation relate to SSC training, three questions based on Steindl, Kirby, and Tellegan (2017) [23] were asked about practicing SSC. Participants were asked to rate their confidence, commitment, and importance in practicing SSC, each on a 10-point sliding scale.

\subsubsection{Engagement and Demographic Variables}

The post-intervention questionnaire included the same questions as the pre-intervention questionnaire and asked participants to estimate their time spent engaging with the SSC training program and time spent practicing SSC. In addition, participants were asked how much they felt seen and heard on a 4-point scale ranging from 1 (not all) to 4 (very much) and how much they used the discussion board on a 10-point scale ranging from 1 (occasionally) to 10 (frequently). Demographic information, including age category, gender identity, and previous SSC or other mindfulness and self-compassion training were also collected.

\section{Results}

The findings of the present study support our prediction that SSC training would significantly improve psychological wellbeing across a variety of measures. Of the 32 participants, seven had previously completed SSC training. When these participants were removed from the analyses all but one (mindfulness subscale, see Table 1 ) of the effects remained significant. All inferential statistics from paired samples $t$-tests reported correspond to the total sample. To be conservative in our testing of multiple dependent variables, a Bonferroni correction for multiple tests was used for all $t$-test comparisons. This shifted the threshold for significance (alpha) from 05 to 01 . Descriptive statistics and effect sizes for all primary dependent variables in both the total sample and the subset that had no previous training are presented in Table 1.

Table 1 Descriptive statistics for primary dependent variables.

\begin{tabular}{lllllllll}
\hline & \multicolumn{4}{c}{ All Participants $(\boldsymbol{N}=\mathbf{3 2})$} & \multicolumn{3}{c}{ No Previous SSC $(\boldsymbol{N}=\mathbf{2 5})$} \\
\hline \multirow{3}{*}{ Perceived Stress } & \multicolumn{3}{c}{$M$} & SD & $d$ & M & SD & $d$ \\
& T1 & 20.76 & 6.52 & & 20.48 & 5.25 & \\
Internalized Shame & 15.94 & 5.98 & $.74 * *$ & 15.92 & 6.36 & $.82^{* *}$ \\
& T1 & 45.09 & 17.27 & & 44.88 & 14.88 & \\
& T2 & 34.28 & 15.64 & $.85^{* *}$ & 32.72 & 14.42 & $1.18^{*}$ \\
\hline
\end{tabular}




\begin{tabular}{|c|c|c|c|c|c|c|c|}
\hline \multirow[t]{2}{*}{ Bodily Shame } & T1 & 10.78 & 3.54 & & 10.60 & 3.42 & \\
\hline & $\mathrm{T} 2$ & 8.86 & 2.98 & $.57^{*}$ & 8.32 & 2.98 & $.82 * *$ \\
\hline \multirow[t]{2}{*}{ Coping Self-Efficacy } & $\mathrm{T} 1$ & 129.72 & 45.19 & & 130.16 & 41.58 & \\
\hline & T2 & 174.94 & 41.33 & $1.17^{* *}$ & 176.44 & 42.92 & $1.27^{* *}$ \\
\hline \multirow[t]{2}{*}{ Self-Compassion } & $\mathrm{T} 1$ & 2.90 & .64 & & 2.92 & .50 & \\
\hline & T2 & 3.41 & .60 & $.95 * *$ & 3.44 & .527 & $.93 * *$ \\
\hline \multirow[t]{2}{*}{ Self-kindness } & $\mathrm{T} 1$ & 3.02 & .86 & & 2.99 & .71 & \\
\hline & T2 & 3.67 & .64 & $.67 * *$ & 3.69 & .65 & $.80^{*}$ \\
\hline \multirow[t]{2}{*}{ Common Humanity } & T1 & 2.92 & .89 & & 3.02 & .71 & \\
\hline & T2 & 3.45 & .86 & $.76 * *$ & 3.51 & .88 & $.69 *$ \\
\hline \multirow[t]{2}{*}{ Mindfulness } & T1 & 3.20 & .83 & & 3.21 & .72 & \\
\hline & $\mathrm{T} 2$ & 3.68 & .82 & $.56^{*}$ & 3.66 & .82 & .51 \\
\hline \multirow[t]{2}{*}{ Self-Judgment } & $\mathrm{T} 1$ & 3.11 & .35 & & 3.11 & .34 & \\
\hline & T2 & 2.86 & .27 & $.89 * *$ & 2.85 & .28 & $.90^{* *}$ \\
\hline \multirow[t]{2}{*}{ Isolation } & T1 & 3.38 & .98 & & 3.41 & .79 & \\
\hline & $\mathrm{T} 2$ & 2.76 & .98 & $.82 * *$ & 2.73 & .87 & $.82 * *$ \\
\hline \multirow[t]{2}{*}{ Over-Identification } & T1 & 3.30 & .96 & & 3.22 & .85 & \\
\hline & T2 & 2.70 & .88 & $.78 * *$ & 2.61 & .78 & $.72 *$ \\
\hline
\end{tabular}

Note. The abbreviations for this table are as follows: T, time; SSC, Somatic Self-Compassion; $M$, mean; $S D$, standard deviation; $d$, Cohen's d effect size. All comparisons are from T1 to T2, ${ }^{* *} p$ $<.001,{ }^{*} p<.01$. The Bonferroni corrected threshold was set at .01 .

Completion of SSC training was associated with significant reductions in perceived stress $t(31)=$ $4.16, p<.001$, internalized shame $t(31)=4.79, p<.001$, and bodily shame $t(31)=3.22, p=.003$. In addition, SSC training resulted in significant increases in self-esteem $t(31)=-4.59, p<.001$, coping self-efficacy $t(31)=-6.63, p<.001$, and overall self-compassion $t(31)=-5.29, p<.001$. The six subscales of self-compassion were also assessed and revealed a significant increase in selfkindness $t(31)=-3.80, p=.001$, common humanity $t(31)=-4.29, p<.001$, and mindfulness $t(31)=$ -3.19, $p=.003$. The negative subscales revealed a significant reduction in self-judgment $t(31)=$ $4.97, p<.001$, isolation $t(31)=4.61, p<.001$, and over-identification $t(31)=4.42, p<.001$.

Participants reported practicing SSC an estimated average of 2.5 hours per week $(S D=1.05$, range $=0.5-4.5$ hours), and engaged with the training materials an estimated average of 2.25 hours per week ( $S D=1.26$, range $=0.5-5$ hours). Average number of sessions attended (live or recorded) was 9.97 ( $S D=2.71$, range $=1-12$ ). Time spent in practice, engaging with training materials and number of sessions attended did not significantly correlate with any of the primary dependent variables at time 2 except for self-judgment and self-kindness. Amount of time engaged with the materials was significantly and negatively correlated with self-judgment $r(30)=-$ $.69, p<.001$. Number of gatherings attended was significantly and positively correlated with selfkindness $r(30)=.38, p<.03$. Discussion board use was moderate throughout the training $(M=$ $5.47, S D=2.72$, range $=0-10)$. Participants reported a high level of feeling seen and heard $(M=$ $3.84, S D=.37$, range $=3-4$ ) throughout the training, which significantly correlated with selfjudgment, coping self-efficacy, perceived stress, and confidence (all $r$ 's $>.35$, all $p^{\prime} s<.05$ ). 
Confidence, importance, and commitment to practice did not differ from time 1 to time 2 (all $t^{\prime} s<1.24$, all $p^{\prime} s>.23$, mean range 8.13 - 9.29). Similarly, these measures did not significantly correlate with any primary dependent variables at time 2 apart from self-compassion. Participants' confidence in their ability to practice SSC at home was significantly and positively correlated with total self-compassion $r(30)=.38, p=.034$ and self-kindness $r(30)=.52, p=.003$ and negatively correlated with and self-judgment $r(30)=-.47, p=.008$. Commitment to practice was also positively correlated with self-kindness $r(30)=.38, p=.032$.

\section{Discussion}

Somatic Self-Compassion ${ }^{\circledR}$ training resulted in significant improvements in a variety of measures of psychological and emotional wellbeing over time. At the end of the training, participants' perceived stress, internalized shame, and bodily shame were all significantly reduced while coping self-efficacy and self-compassion were significantly increased. Within the subscales of self-compassion, self-judgment, isolation, and over-identification were all significantly reduced and self-kindness, common humanity, and mindfulness were all significantly increased. All effect sizes were moderate to strong (Table 1). These initial findings suggest that training in SSC for 13 weeks can create significant shifts in participants' felt and perceived experiences, resulting in a greater sense of wellbeing.

Our findings are consistent with findings from other comparable compassion trainings. Based on a recent meta-analysis of randomized controlled trials (RCTs), outcome categories within compassion-based interventions include compassion, self-compassion, mindfulness, depression, anxiety, psychological distress and wellbeing [32]. A consistent finding is a reduction of psychological distress, anxiety, and depression and an improvement in compassion, selfcompassion, mindfulness, and wellbeing. Of the interventions included and as mentioned previously, MSC [18] is most closely aligned with SSC training. In their feasibility study and RCT, Neff and Germer [18] found that MSC training in a non-clinical sample led to a significant reduction in perceived stress and gains in self-compassion, using the same operationalizations as the present study. While effect sizes were not reported $(N=23)$ in the feasibility study, they found a $35 \%$ increase in self-compassion and a $13 \%$ reduction in perceived stress, while the present study $(N=32)$ found an $18 \%$ increase in self-compassion and a $24 \%$ reduction in perceived stress. Thus, the effects of SSC training on stress/distress and self-compassion are consistent with MSC, though the SSC focus on coping with stress through self-compassion may be evident in the greater reduction in perceived stress and the smaller gains in self-compassion. Indeed, the efficacy of SSC in promoting participants' beliefs that they can cope with difficult situations is supported by our robust increase $(d=1.17)$ in coping self-efficacy.

Identification of the ability of SSC training to significantly reduce internalized and bodily shame is relatively unique to this study. While we were unable to find studies that specifically examined the effects of self-compassion training on bodily shame from the Experience of Shame Scale, we chose to include this measure because of the somatic focus of SSC. Discussions of shame related to participants' bodies have consistently occurred throughout the trainings and we wanted to get a pulse on whether SSC was able to reduce the experience of bodily shame, or whether the somatic focus tended to highlight it. With a moderate reduction $(d=.57)$ in bodily shame reported, we conclude that these effects are both beneficial and warrant further investigation. 
Interestingly, at the time of writing, it appears that only a couple of self-compassion studies have examined internalized (trait) shame as a dependent variable. In a pre-trial of Compassionate Mind Training [21], a small sample $(N=6)$ of clinical patients showed significant reductions in external shame (Other as Shamer Scale, which the authors state was developed from the Internalized Shame Scale). Au et al [19] examined the effects of compassion-based therapy in a small sample $(N=10)$ of women with PTSD and found significant reductions in internalized shame and improvements in self-compassion. The present study, with a notable reduction $(d=.85)$ in internalized shame adds weight to the evidence that self-compassion training, particularly in a non-clinical population, can significantly reduce internalized (trait) shame.

Together with the present findings we may conclude that self-compassion training is a promising intervention to consider when a reduction in various types of shame is a desired outcome. Indeed, others suggest that self-compassion is the antithesis to internalized shame and that reduction in shame may be the mechanism for how self-compassion improves psychosocial outcomes ([33]; specifically looking at people living with HIV). This has particular relevance for individuals who have experienced traumatic stress (diagnosed with PTSD or not) given the strong relationship between shame and trauma symptoms $[19,20]$.

The primary limitation of this study is that it is a pre-post design with no waitlist or active control group. Conducting an RCT would be the next step to assess the efficacy of SSC training. In their meta-analysis, Kirby et al. [32] identified that in this new field of research, the number of RCTs are still lacking. This is understandable, given the time and cost required of each iteration of study to go from a feasibility study to waitlist control and active control comparison. In fact, Kirby et al. [32] was only able to identify 4 studies with active controls, 17 with waitlist controls, and had to exclude 34 studies that did not include a comparison group. The lack of a comparison group prevents us from ruling out alternative explanations for our findings, including maturation or social desirability bias. In addition, the positive effects of SSC training shown in the present study could be influenced by selection bias, in that the self-selection to participate may have resulted in only highly motivated participants taking part in the study. This is supported by the high level of motivation (confidence, importance and commitment to practice) measured in the current participants.

The present study's pre-post design is strengthened in that it includes participants across five separate cohorts over the span of 14 months all with the same instructor. In addition, our preintervention assessment occurred after community was established within the SSC training (week 2-3) making our baseline data less likely to be impacted by extreme values. Together, our design allows us to reasonably exclude the possibility of robust regression to the mean or time- and cohort-linked variables that could account for our findings. It should also be noted that the presence of the instructor could be a moderating variable in the efficacy of SSC training and that our sample size $(N=32)$ remains small. Future studies looking at self-led iterations of SSC training could shed light on the impact of the instructor presence as well as the role of motivation.

\section{Conclusions}

The findings of this feasibility study indicate that SSC training is on par with other previously established and empirically-supported self-compassion trainings in that it provides motivated individuals with the knowledge, skills, and support to reduce stress and shame while increasing 
coping self-efficacy and self-compassion. The increased focus on somatic intelligence (i.e., interoception and sensory modulation) as well as being intentionally trauma-informed when combined with the reductions in shame make SSC training a good fit for sub-clinical populations of trauma survivors. In the context of moving the field of compassion-based interventions forward, the present study adds strength to the case for continued inclusion of shame as a dependent variable in order to understand the relationship between self-compassion, shame, and stress.

\section{Author Contributions}

Study design, data collection, analysis, and interpretation were conducted by Sara B Taylor. Kristy Arbon and Faith Reynolds provided literature sources, and writing assistance with the introduction, materials, and discussion sections. Kristy Arbon also recruited participants and served as the instructor for all cohorts.

\section{Funding}

This research was funded by the office of Academic Affairs and the Psychology Department at Hendrix College.

\section{Competing Interests}

Kristy Arbon is the owner of HeartWorks Training LLC, and the creator and instructor for the Somatic Self-Compassion ${ }^{\circledR}$ program described in this research.

\section{References}

1. Yang TC, Matthews SA, Chen VY. Stochastic variability in stress, sleep duration, and sleep quality across the distribution of body mass index: Insights from quantile regression. Int J Behav Med. 2014; 21: 282-291.

2. Shields GS, Slavich GM. Lifetime stress exposure and health: A review of contemporary assessment methods and biological mechanisms. Soc Personal Psychol Compass. 2017; 11: e12335.

3. McEwen BS, Sapolsky RM. Stress and cognitive function. Curr Opin Neurobiol. 1995; 5: 205216.

4. Feeney J, O'Sullivan M, Kenny RA, Robertson IH. Change in perceived stress and 2-year change in cognitive function among older adults: The Irish longitudinal study on ageing. Stress Health. 2018; 34: 403-410.

5. Kilpatrick DG, Resnick HS, Milanak ME, Miller MW, Keyes KM, Friedman MJ. National estimates of exposure to traumatic events and PTSD prevalence using DSM-IV and DSM-5 criteria. J Trauma Stress. 2013; 26: 537-547.

6. Liquido K. Get better at handling stress by fighting these 10 unhealthy habits [Internet]. South San Francisco: Verily; 2016 October 11. Available from: https://verilymag.com/2014/10/unhealthy-habits-how-to-handle-stress.

7. Rodriquez EJ, Gregorich SE, Livaudais-Toman J, Pérez-Stable EJ. Coping with chronic stress by unhealthy behaviors: A re-evaluation among older adults by race/ethnicity. J Aging Health. 2017; 29: 805-825. 
8. Mehling WE, Chesney MA, Metzler TJ, Goldstein LA, Maguen S, Geronimo C, et al. A 12-week integrative exercise program improves self-reported mindfulness and interoceptive awareness in war veterans with posttraumatic stress symptoms. J Clin Psychol. 2018; 74: 554-565.

9. Paulus MP, Flagan T, Simmons AN, Gillis K, Kotturi S, Thom N, et al. Subjecting elite athletes to inspiratory breathing load reveals behavioral and neural signatures of optimal performers in extreme environments. PloS ONE. 2012; 7: e29394.

10. Van der Kolk Ba. The body keeps the score: Brain, mind, and body in the healing of trauma. New York: Viking; 2014.

11. Fisher J. Sensorimotor psychotherapy in the treatment of trauma. Pract Innov. 2019; 4: 156165.

12. Champagne T. Sensory modulation and environment: Essential elements of occupation. 3rd ed. Sydney: Pearson; 2011.

13. Brown A, Tse T, Fortune T. Defining sensory modulation: A review of the concept and a contemporary definition for application by occupational therapists. Scand J Occup Ther. 2018; 26: 515-523.

14. Mahler K. Interoception: The eighth sensory system: Practical solutions for improving selfregulation, self-awareness and social understanding of individuals with autism spectrum and related disorders. Shawnee: AAPC Publishing; 2016.

15. Zessin U, Dickhäuser $O$, Garbade S. The relationship between self-compassion and well-being: A meta-analysis. Appl Psychol Health Well Being. 2015; 7: 340-364.

16. Rockliff H, Gilbert P, McEwan K, Lightman S, Glover D. A pilot exploration of heart rate variability and salivary cortisol responses to compassion-focused imagery. 2008; 5: 132-139.

17. Breines JG, Thoma MV, Gianferante D, Hanlin L, Chen X, Rohleder N. Self-compassion as a predictor of interleukin- 6 response to acute psychosocial stress. Brain Behav Immun. 2014; 37: 109-114.

18. Neff KD, Germer CK. A pilot study and randomized controlled trial of the mindful self-compassion program. J Clin Psychol. 2013; 69: 28-44.

19. Au TM, Sauer-Zavala S, King MW, Petrocchi N, Barlow DH, Litz BT. Compassion-based therapy for trauma-related shame and posttraumatic stress: Initial evaluation using a multiple baseline design. Behav Ther. 2017; 48: 207-221.

20. La Bash H, Papa A. Shame and PTSD symptoms. Psychol Trauma. 2014; 6: 159-166.

21. Jazaieri H, Jinpa GT, McGonigal K, Rosenberg EL, Finkelstein J, Simon-Thomas E, et al. Enhancing compassion: A randomized controlled trial of a compassion cultivation training program. J Happiness Stud. 2013; 14: 1113-1126.

22. Gilbert $P$, Procter $S$. Compassionate mind training for people with high shame and self-criticism: Overview and pilot study of a group therapy approach. Clin Psychol Psychother. 2006; 13: 353-379.

23. Steindl SR, Kirby JN, Tellegan C. Motivational interviewing in compassion-based interventions: Theory and practical applications. Clin Psychol. 2018; 22: 265-279.

24. SAMHSA's Trauma and Justice Strategic Initiative. SAMHSA's Concept of Trauma and Guidance for a Trauma-Informed Approach. Rockville: Substance Abuse and Mental Health Services Administration; 2014.

25. Cohen S, Kamarck T, Mermelstein R. A global measure of perceived stress. J Health Soc Behav. 1983: 385-396. 
26. Cook DR. Measuring shame: The internalized shame scale. Alcohol Treat Q. 1988; 4: 197-215.

27. Rosenberg M. Society and the adolescent self-image. Princeton: Princeton University Press; 1965.

28. Andrews B, Hunter E. Shame, early abuse, and course of depression in a clinical sample: A preliminary study. Cogn Emot. 1997; 11: 373-381.

29. Andrews B, Qian M, Valentine JD. Predicting depressive symptoms with a new measure of shame: The experience of shame scale. Br J Clin Psychol. 2002; 41: 29-42.

30. Chesney MA, Neilands TB, Chambers DB, Taylor JM, Folkman S. A validity and reliability study of the coping self-efficacy scale. Br J Health Psychol. 2006; 11: 421-437.

31. Neff KD. The development and validation of a scale to measure self-compassion. Self Identity. 2003; 2: 223-250.

32. Kirby JN, Tellegen CL, SteindI SR. A meta-analysis of compassion-based interventions: Current state of knowledge and future directions. Behav Ther. 2017; 48: 778-792.

33. Williams SL, Fekete EM, Skinta MD. Self-compassion in PLWH: Less internalized shame and negative psychosocial outcomes. Behav Med. 2019; 45: 1-9.

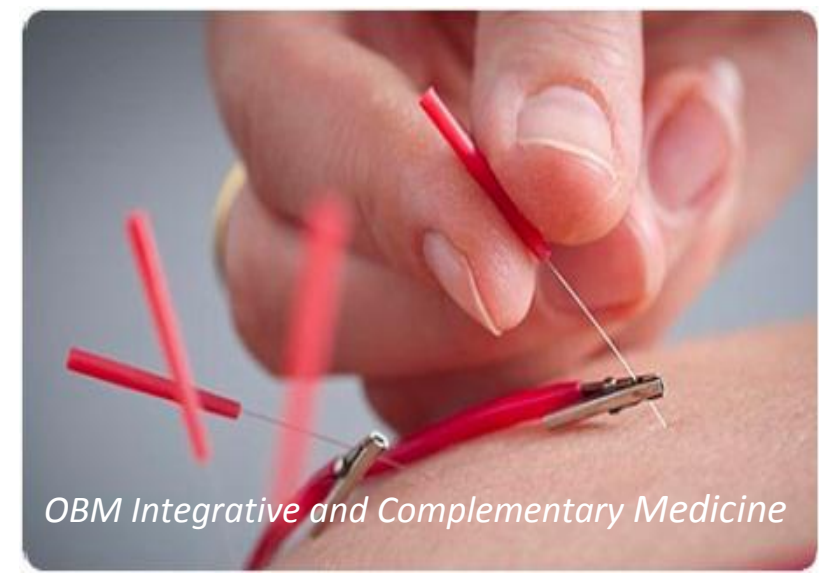

Enjoy OBM Integrative and Complementary Medicine by:

1. Submitting a manuscript

2. Joining in volunteer reviewer bank

3. Joining Editorial Board

4. Guest editing a special issue

For more details, please visit:

http://www.lidsen.com/journals/icm 